\title{
Assessment of antimicrobial effectiveness of natural dyed fabrics
}

\author{
S. Datta ${ }^{1}$, M. A. Uddin ${ }^{2}$, K. S. Afreen ${ }^{1}$, S. Akter ${ }^{1}$ and A. Bandyopadhyay ${ }^{3}$ \\ ${ }^{1}$ Dept. of Microbiology, Primeasia University, HBR Tower, 9 Banani, Dhaka 1213, Bangladesh \\ ${ }^{2}$ Dept. of Textile Engineering, Primeasia University, Star Tower, 12, Kamal Ataturk Avenue, Banani, Dhaka \\ 1213, Bangladesh \\ ${ }^{3}$ DOEACC Centre, Jadavpur University Campus, Kolkata-700032, India
}

\begin{abstract}
There is an increasing interest in adding value to textiles by the use of natural products. Many of the plant materials, from which natural dyes are obtained, found to have some medicinal values. In the current study, dyeing materials were prepared from pomegranate (Punica granatum), wild mangosteen (Diospyros peregrine), myrabalan (Terminalia chebula), arjun (Terminalia arjuna), betel nut (Areca catech), onion (Allium cepa), tea (Camellia sinensis), neem (Camellia sinensis), eucalyptus (Eucalyptus cinerea) and dye flower (Coreopsis basalis). Cotton fabrics were dyed with the extracted colouring materials and were tested for their wash fastness to ensure the durability of the colour on the fabrics. Finally, the antimicrobial property of ten different natural dyed fabrics was tested against Bacillus subtilis (Gram positive) and Escherichia coli (Gram negative). The cotton fabrics dyed with extracts of arjun, betel nut, pomegranate, tea and onion were found to have antimicrobial activity against both the test bacteria at varying efficiency. The dyed fabrics also showed reasonably good wash fastness; hence have practical potential for adding antibacterial properties along with vibrant colours to textiles of medical and other delicate uses.
\end{abstract}

Keywords: Antimicrobial effectiveness; Betel nut; Cotton fabric; Natural dye; Pomegranate; Tea

\section{Introduction}

Dyeing is an ancient art, which predates written records. Primitive dyeing techniques included sticking plants to fabric or rubbing crushed pigments onto cloth. Techniques of using natural dyes and increasing water fastness (resistance) were then evolved. Up to the end of the 19th century, natural dyes were the main colorants for textiles. Presently, synthetic dyes have replaced the natural dyes due to the properties e.g. wide range of colors, higher reproducibility and improved quality of the textiles which can be achieved by lower unit cost (Bechtold et al., 2006).

Ecological considerations are becoming important factors in the selection of consumer goods all over the world. Production and application of synthetic dyes release vast amount of waste and unfixed colorants poses serious health hazard and are disturbing the eco-balance of the nature. During the mid 1980s, more interest have been shown in the use of natural dyes as it is believed that these primitive dyes are less toxic, less allergic and more eco-friendly than the synthetic ones (Mehrabian et al., 2000). But the problems associated in dying with natural dyes are lower extraction of natural colorants and poor fastness. To overcome these problems various metallic salts are being used as mordant, which are traditionally used to improve fastness and produce different shades with the same dye (Cristea and Vilarem, 2006, Hwang et al., 1998, Lee and Kim, 2004 and Shin and Choi, 1999).

There has been increasing interest in building antibacterial properties into textiles. Clothing can act as carriers for both the pathogenic and odor generating bacteria or moulds by offering an ideal environment for microbial attachment and growth by providing oxygen, water and warmth, as well as nutrients from spillages and body exudates. Antibacterial finishes are also highly desirable for textiles, which are to be used in hospitals and hotels. Textiles are liable to promote cross infection and promote disease; in fact hygiene problems with hospital textiles directly interfere with the recovery of patients (Gupta et al., 2004). Many of the plants traditionally used in dyeing are credited with medicinal properties. For example, a commonly used natural dye source,

\footnotetext{
*Corresponding author. e-mail: suvamoy.datta@primeasia.edu.bd
} 
pomegranate (Punica granatum) is rich in hydrolysable tannins, a class of compounds known to exhibit a remarkable degree of antimicrobial activity (Hussein et al., 1997). A number of other plant dye sources are rich in naphthaquinones, including lawsone from henna, juglone from walnut, and lapachol from alkanet, which have already been reported to comprise antibacterial, antifungal, antiviral and antineoplasic activity (Lagrota et al., 1986; Schuerch, 1978 and Wagner et al., 1989).

In the current investigation, the antimicrobial activity of some of the natural dyeing agents available in Bangladesh has been examined. Should the dyes themselves be inherently antimicrobial, therapeutic and medical textiles could be produced at no additional cost. The dyed fabrics have been tested for their wash fastness also to ensure the durability of the antimicrobial effect. In this study, E. coli and Bacillus subtilis were used as representatives for Gram positive and Gram negative bacteria.

\section{Materials and methods}

\section{Preparation of mordant}

For the experiment we used alum (aluminum potassium sulfate) as a mordant. Too much use of alum as a mordant makes the yarn sticky. To help offset this possibility; alum is usually combined with cream of tartar (Weigle, 1974). We prepared $5 \%$ solution of alum in hot water as mordant.
Preparation of dyes

Bangladesh has a rich repository of dye producing plants. For our study we selected ten different plant materials. The list of the plant species, their used parts and resulting colour has been given in Table I. The respective plant materials were dried and crushed into small flakes; $50 \mathrm{~g}$ of each was added to $1000 \mathrm{ml}$ of water, boiled at $60-70{ }^{\circ} \mathrm{C}$ in a closed bath for $1 \mathrm{~h}$. Then the liquor was filtered and stored. The valid time of the liquor is $6 \mathrm{~h}$.

\section{Fabric pre treatment}

A plain weave cotton fabric was used. At first, the fabric was cleansed thoroughly off starch and other impurities. The cotton fabric was boiled for $20 \mathrm{~min}$ in a solution containing $4 \%$ liquid soap, $8 \%$ soda ash and $2 \%$ caustic soda and was then washed off in clean water and soaked in a 3\% solution of bleaching powder for 5-10 min. The fabric was given a final rinse in cold water.

\section{Mordant and dyeing}

The prepared mordant was mixed into the dyeing solutions, stirring constantly using wooden rods for even dyeing. The concentrations and volume of mordant-dye mixture will depend on the fabric (texture, thickness and area) and the color intensities desired. In this study, $5.0 \mathrm{~g}$ of fabric to be

Table I. List of plants materials used for dyeing of cotton fabrics and corresponding colour obtained in the study

\begin{tabular}{llcc}
\hline Plant materials & Botanical Name & Part Used & Colour of dyed fabric \\
\hline Pomegranate & Punica granatum & rind & khaki \\
Wild Mangosteen & Diospyros peregrine & fruit & gray / pink \\
Myrabalan & Terminalia chebula & fruit & gray / gold \\
Arjun & Terminalia arjuna & saw dust & mauve pink \\
Betel nut & Areca catechu & nut & deep pink \\
Onion & Allium cepa & skin & pink \\
Tea & Camellia sinensis & leaves & beige \\
Neem & Azadirachta indica & leaves & light green \\
Dye flower & Coreopsis basalis & leaves & brown \\
Eucalyptus & Eucalyptus cinerea & leaves & gold and gray \\
\hline
\end{tabular}


dyed was put in $160 \mathrm{ml}$ of warm alum solution for $30 \mathrm{~min}$. After squeezing, the sample was put into $200 \mathrm{ml}$ of dyeing liquor for $1 \mathrm{~h}$ at $60^{\circ} \mathrm{C}$. For control fabric, similar fabric swatch was put into $160 \mathrm{ml}$ of mordant, treated as it was done for test fabrics, and then put into $200 \mathrm{ml}$ of autoclaved distilled water instead of dyeing liquor for $1 \mathrm{~h}$ at $60^{\circ} \mathrm{C}$. The dyed fabrics as well as control fabrics were washed thoroughly in liquid soap and plenty of cold water till the water ran crystal clear.

\section{Wash fastness}

Wash fastness of dye is influenced by the rate of diffusion of dye and state of dye inside the fiber. The dyed fabrics were tested for their wash fastness to ensure the durability of the color of the dyeing material on the fabric. The wash-fastness properties of the dyed fabrics were measured according to ISO standard recipe using spectrophotometer. After dyeing, the swatches were rinsed and washed with soft water by using Gemsan washing substance as described follows: rinsing twice with cold water, adjusting to $\mathrm{pH} 5$ to 6 with acetic acid, washing for $20 \mathrm{~min}$ in hot boiling water with ECE detergent (a non phosphate detergent manufactured for use in ISO 6330 and in the ISO $105 \mathrm{C}$ series of colour fastness tests. The powder is free from fluorescent brightening agent), rinsing once with warm water and rinsing three times with cold water or until seeing no colorant in rinsing water. Instrumental measurements of fastness were made with spectrophotometer Filter Wheel CE, Spectraflash SF600X®, USA at $600 \mathrm{~nm}$ of wavelength according to ISO standards.

\section{Study of antimicrobial effectiveness}

Circular swatches of $4.8 \pm 0.1 \mathrm{~cm}$ ( $1.9 \pm 0.03$ inches) in diameter were cut from the test dyed fabrics. The swatches were stacked in a $250 \mathrm{~mL}$ wide mouthed glass jar with screw cap. Similarly swatches of the control mordant treated fabric with same fiber type and fabric construction were taken as control test samples. The swatches were placed separately in sterile petri dishes and a standardized microbial sample containing $10^{8} \mathrm{cfu} / \mathrm{ml}$ of the test organisms (B. subtilis and E. coli) prepared in normal saline $(0.9 \% \mathrm{NaCl})$ were inoculated ensuring uniform distribution of the inoculum. These swatches were transferred aseptically to the jar and covered tightly to prevent evaporation. Control and test fabric swatches are inoculated with microorganisms. The inoculation is per- formed such that the microbial suspension touches only the fabric. Bacteria levels on both control and test fabrics are determined at "time zero" by elution in a large volume of neutralizing broth, followed by dilution and plating. A control is run to verify that the neutralization/elution method effectively neutralizes the antimicrobial agent in the fabric. Additional inoculated control and test fabrics are allowed to incubate, undisturbed in sealed jars, for 24 hours. After incubation, microbial concentrations are determined. Reduction of microorganisms relative to initial concentrations and the control fabric is calculated. The procedure described in detail in AATCC TM100, which has been designed to quantitatively test the ability of fabrics and textiles to inhibit the growth of microorganisms or kill them, over a 24 hour period of contact. Finally, after incubation, bacterial counts were reported and percentage (\%) of antimicrobial potency of natural dyes against Gram positive and Gram negative bacteria were determined, by the formula, $\mathrm{R}=100 \times(B-A) / B$ (AATCC, 2006); where: $R=\%$ reduction, $A=$ the number of bacteria recovered from the inoculated treated test specimen swatches in the jar incubated over the desired contact period, $B=$ the number of bacteria recovered from the inoculated treated test specimen swatches in the jar immediately after inoculation (at "0" contact time).

\section{Results and discussion}

Dyed fabrics of various colours were obtained by using different natural agents. Respective colour development of the dyed fabrics is shown in Fig. 1. The wash fastness of the dyed fabrics was measured according to the ISO standard recipe using Spectrophotometer. Dyes of arjun showed excellent color and staining fastness followed by myrabalan, wild mongosteen, betel nut, tea, dye flower and onion. Pomegranate, eucalyptus and neem were found to show average color and staining fastness.

Natural dyed fabric is environmental friendly product as there is no use of additional chemicals in the dyeing process except mordant. Ten fabric samples of the alum mordanted dyes were used for the antimicrobial test. Out of the 10 dyed fabric samples, 5 samples (Table II) were found to show some extent of inhibitory effect on B. subtilis (a gram positive bacteria) and 6 samples showed some extent of inhibitory effect on $E$. coli (a gram negative bacteria). The natural 


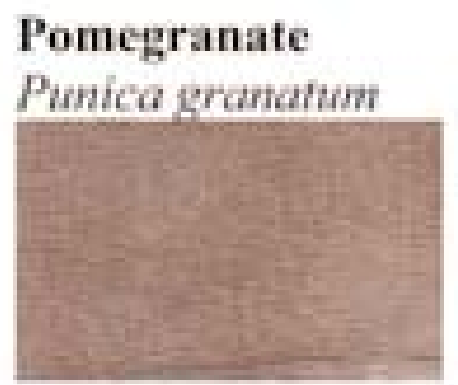

Wild mangosteen

Diospyros peregrine

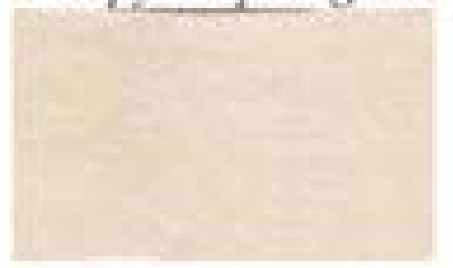

Myrabalan

Terminalia chebula

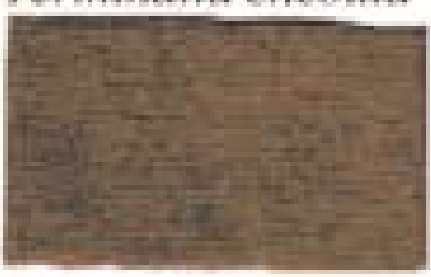

Arjun

Teminalla dring

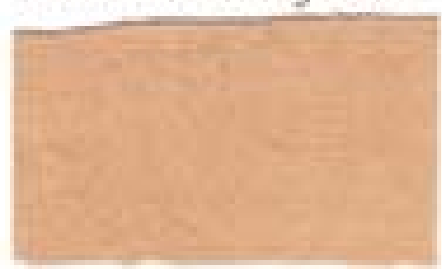

Betel nut

Areca coltechu

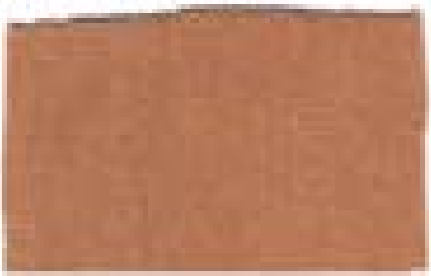

Onion

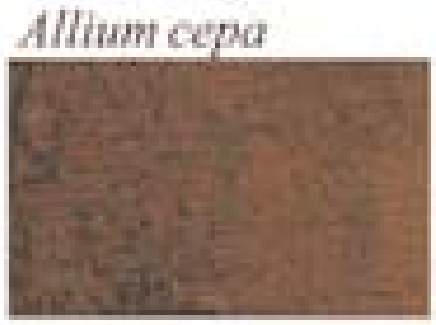

Ten

Canellia sinensis:

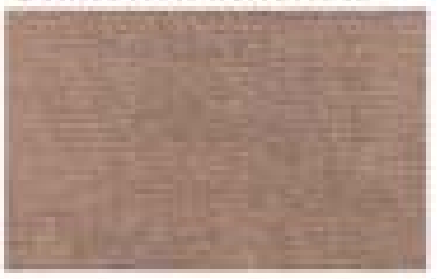

Neem

Anodiracha hilca

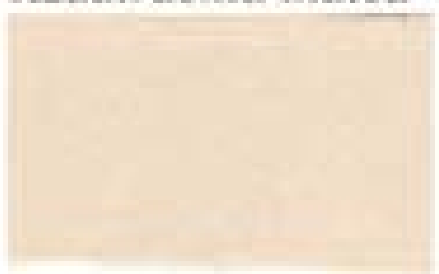

Eucaluptus

Eucalyph cherea

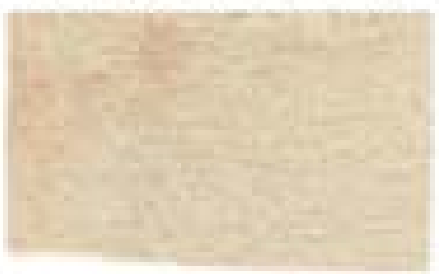

Dye-flower

Corcopsis basalis

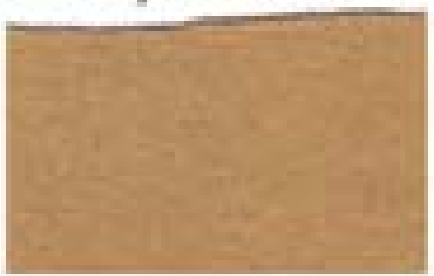

Fig. 1. The corresponding colour of cotton (100\%) fabrics obtained from dyeing with different plant materials after checking wash fastness 
dyed fabrics obtained from arjun, betel nut, pomegranate, tea and onion showed antimicrobial activity against both gram positive and gram negative bacteria. Only myrabalan showed antimicrobial action against gram negative bacteria. Among the ten natural dyed fabrics, arjun was found to be the best, in the present study, which could destroy both the gram positive (85\%) and gram negative bacteria $(70 \%)$ in a significant number. As a result, the user can get antimicrobial potency from these types of natural dyed fabrics. Natural dyed textile goods also have more durability because of its properties of antimicrobial potency which protect the fabric from reducing of the fiber strength and luster.

A question about the contribution of the inherent antibacterial activity of mordant on the total observed antimicrobial activity might come to one's mind. But the subsequent washing and steps involving detection of wash fastness has successfully removed the inhibitory effect of mordant. Still, the antimicrobial effect of control fabric swatch was taken consideration as negative control to calculate the antimicrobial effect of natural dyed fabrics. As shown in Table II, the fabrics dyed by the extracts prepared from parts of dye-flower, neem, eucalyptus and wild mangosteen did not show any

Table II. Analysis of antimicrobial potency of various natural dyed fabrics

\begin{tabular}{lcc}
\hline Sample Name & \multicolumn{2}{c}{$\begin{array}{c}\text { Anti microbial potency (\%) } \\
\text { of natural dyed fabrics } \\
\text { against test organisms }\end{array}$} \\
\hline Tea & B. subtilis & E. coli \\
Dye-Flower & 90 & 50 \\
Myrabalan & 0 & 0 \\
Neem & 0 & 40 \\
Pomegranate & 0 & 0 \\
Betel nut & 90 & 55 \\
Eucalyptus & 80 & 60 \\
Arjun & 0 & 0 \\
Wild Mangosteen & 05 & 70 \\
Onion & 30 & 0 \\
\hline
\end{tabular}

The procedure in AATCC TM100 (AATCC, 2006) was followed for determining the percentage of antimicrobial potency of the natural dyes. antimicrobial effectiveness against any of the test bacteria, which clearly indicating that the antimicrobial effect observed was exclusively due to the respective natural dye. Arjun, betel nut, pomegranate, tea and onion were able to reduce the growth of both Gram positive and -negative bacteria, which clearly indicates that these dyes have broad range of antimicrobial activity. All among them showed comparatively greater resistance to Gram positive bacteria except for onion showing similar activity against both Gram positive and negative bacteria. Myrabalan showed antibacterial effect against Gram negative bacteria only. Since the dyes showed reasonably good wash fastness, it may be assumed that the antimicrobial effect will be durable in practical usage. In practice, there is an exciting opportunity for the use of natural dyes for adding antibacterial properties to clothing and to the textiles used in the medical and hospital uses, preparing clothing for infants and patients allergic to chemical dyes.

\section{Acknowledgement}

We would like to thank Md. Jasim Uddin, Md.Shafiul Islam, Md.Rajib-ul- Bari, S.M. Raship Ahmad, Sanjoy Kumar Ghosh and Md.Zahidul Islam, the final year students of Department of Textile Engineering, Primeasia University for collecting the dyeing materials used in this study.

\section{References}

AATCC Technical Manuals (2006), Quantitative test. TM 100-2004: 150.

Bechtold T, Mussak R, Amalid MA, Ganglberger and E. Geissler S (2006), Extraction of natural dyes for textile dyeing from colored plant wastes released from the food and beverage industry. J. Sci. Food Agri. 86: 233242.

Cristea D and Vilarem G (2006), Improving Light Fastness of Natural Dyes on Cotton Yarn. Dyes Pigments. 70: 238-245.

Gupta D, Khare SK and Laha A (2004), Antimicrobial properties of Natural dyes against Gram-negative bacteria. Colour Technol. 120: 167-171. 
Hussein SAM, Barakat HH, Merfort I and Nawwar MAM (1997), Tannins from leaves of Punica granatum. Phytochem. 45 : 819-823.

Hwang EK, Kim MS, Lee DS and Kim KB (1998), Color development of natural dyes with some mordants. $J$. Korean Fiber Soc. 35: 490-497.

Lee YH and Kim HD (2004), Dyeing properties and color fastness of cotton and silk fabrics dyed with Cassia tora L. extract. Fiber Polymers. 5: 303-308.

Mehrabian S, Majd A and Majd I (2000), Antimicrobial effects of three plants (Rubia tinctorum, Carthamus tinctorius and Juglans regia) on some airborne microorganisms. Aerobiologia. 16 (3): 455-458.

Pinto AV, Pinto Mde C, Lagrota MH, Wigg MD and Aguiar AN (1987), Antiviral activity of naphthoquinones. I.Lapachol derivatives against enteroviruses. Rev. Latinoam. Microbiol. 29 (1): 15-20.
Shin YS and Choi H (1999), Characteristics and dyeing properties of green tea colorants. J. Korean Soc. Clothing Textiles. 23: 385-390.

Schuerch AR (1978), $\beta$-Lapachone, an Inhibitor of Oncornavirus Reverse Transcriptase and Eukaryotic DNA Polymerase- $\alpha$. Eur. J. Biochem. 84: 197-205.

Wagner H, Kresher B, Lotter H, Hamburger MO and Cordell GA (1989), Structure determination of new isomeric Naphtho [2,3-b] furan-4,9-diones from Tabebuia avellanedae by the selective-INEPT technique. Helv. Chim. Acta. 72: 659-667.

Weigle P (1974), Ancient dyes for modern weavers. (Watson- Guptill Publications, New York) 1974.

Received: 23 January 2013; Revised: 04 June 2013; Accepted: 08 July 2013. 